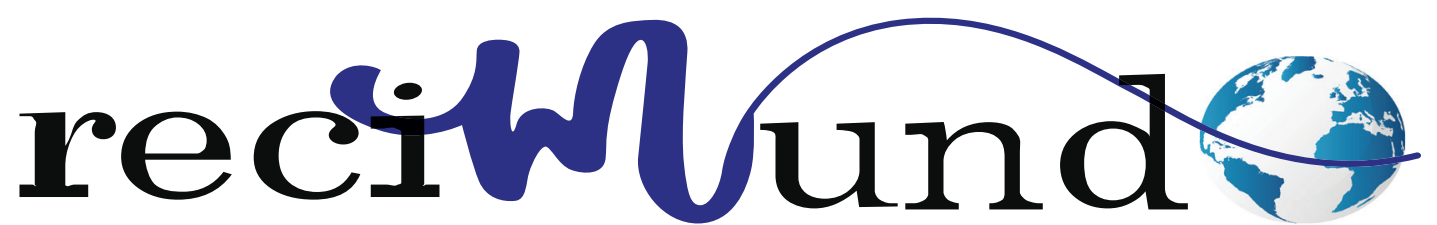

Revista Científica Mundo de la Investigación y el Conocimiento

DOI: $10.26820 /$ recimundo/5.(4).oct.2021.200-206

URL: https://recimundo.com/index.php/es/article/view/1306

EDITORIAL: Saberes del Conocimiento

REVISTA: RECIMUNDO

ISSN: 2588-073X

TIPO DE INVESTIGACIÓN: Reporte de caso

CÓdigo UNESCO: 32 Ciencias Médicas

PAGINAS: 200-206

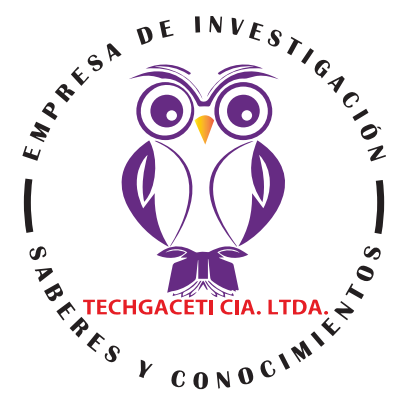

\title{
Agenesia de arteria pulmonar. A propósito de un caso en Hospital Clínica San Francisco
}

Pulmonary artery agenesis. About a case at Hospital Clínica San Francisco Agenesia da artéria pulmonar. Sobre um caso no Hospital Clínica San Francisco

Héctor Enrique Delgado Zambrano'; Mili Alejandra Abad Idrovo2; José Antonio Ledesma Polo3; June Dennisse Bohórquez Garces ${ }^{4}$

RECIBIDO: 02/09/2021 ACEPTADO: 20/09/2021 PUBLICADO: 30/10/2021

1. Médico Cirujano de la Universidad Técnica de Manabí; Investigador Independiente; Guayaquil, Ecuador; hectorzam23@ hotmail.com; (iD https://orcid.org/0000-0001-6301-9284

2. Médico de la Universidad de Guayaquil; Investigador Independiente; Guayaquil, Ecuador; mili_abad@hotmail.com; https://orcid.org/0000-0002-0510-9676

3. Médico de la Universidad de Guayaquil; Investigador Independiente; Guayaquil, Ecuador; josexIp_89@hotmail.com; https://orcid.org/0000-0002-4700-7985

4. Máster Universitario en Dirección y Gestión Sanitaria; Medico; Investigador Independiente; Guayaquil, Ecuador; dbogar547@hotmail.com; iD https://orcid.org/0000-0002-4093-8189

CORRESPONDENCIA

Héctor Enrique Delgado Zambrano

hectorzam23@hotmail.com

Guayaquil, Ecuador

(C) RECIMUNDO; Editorial Saberes del Conocimiento, 2021 


\section{RESUMEN}

La agenesia de la arteria pulmonar, es una malformación y patología rara e infrecuente que tiene una prevalencia de 1 de cada 200 - 300 mil individuos. Es más frecuente su aparición del lado derecho, su diagnóstico se da en los primeros años de vida, ya que es muy infrecuente en la edad adulta y pude ser asintomática o presentar síntomas más asociados a cardiopatías isquémicas. Se presenta caso clínico de paciente masculino 44 años edad, que llega a centro médico con cuadro clínico de 6 horas de evolución, caracterizado por dolor precordial tipo opresivo de gran intensidad que irradia a brazo izquierdo y cuello ipsilateral, además se acompaña de disnea que progresa de medianos a mínimos esfuerzos con náuseas y vómito, se realiza radiografía de tórax y ecocardiograma no encontrándose ninguna anomalía, dándose de alta. Días después paciente regresa con problemas respiratorios para lo cual se reingresa volviéndose a realizar estudios de ecocardiograma. En conclusión, se trató de un caso de agenesia de arteria pulmonar izquierda leve, asociada por una cardiopatía isquémica, diagnosticada por ecocardiograma. Hallazgo infrecuente dentro de la literatura tanto por la localización como por la edad del paciente.

Palabras clave: Pulmonar, Unilateral, Radiografía, Tórax, Agenesia.

\section{ABSTRACT}

Pulmonary artery agenesis is a rare and infrequent malformation and pathology that has a prevalence of 1 in $200-300$ thousand individuals. Its appearance is more frequent on the right side, its diagnosis is given in the first years of life, since it is very infrequent in adulthood and it can be asymptomatic or present symptoms more associated with ischemic heart disease. A clinical case of a 44-year-old male patient is presented, who arrives at the medical center with a clinical picture of 6 hours of evolution, characterized by oppressive chest pain of great intensity that radiates to the left arm and ipsilateral neck, also accompanied by dyspnea that progresses From medium to minimal efforts with nausea and vomiting, a chest x-ray and echocardiogram were performed, no abnormalities were found, and the patient was discharged. Days later, the patient returned with respiratory problems, for which he was readmitted, performing echocardiography studies again. In conclusion, it was a case of mild left pulmonary artery agenesis, associated with ischemic heart disease, diagnosed by echocardiography. An infrequent finding in the literature both due to the location and the age of the patient.

Keywords: Pulmonary, Unilateral, Radiography, Thorax, Agenesis.

\section{RESUMO}

A agenesia da artéria pulmonar é uma malformação e patologia rara e infrequente, com prevalência de 1 em 200 - 300 mil indivíduos. Seu aparecimento é mais frequente no lado direito, seu diagnóstico é dado nos primeiros anos de vida, pois é muito raro na idade adulta e pode ser assintomático ou apresentar sintomas mais associados a cardiopatia isquêmica. É apresentado o caso clínico de um paciente do sexo masculino, 44 anos, que chega ao centro médico com quadro clínico de 6 horas de evolução, caracterizado por dor torácica opressora de grande intensidade que se irradia para o braço esquerdo e pescoço ipsilateral, também acompanhada de dispneia que progride De médios a mínimos esforços com náuseas e vômitos, foi realizada radiografia de tórax e ecocardiograma, nenhuma anormalidade foi encontrada e o paciente teve alta hospitalar. Dias depois, o paciente retornou com problemas respiratórios, pelo qual foi readmitido, realizando novamente estudos ecocardiográficos. Concluindo, tratava-se de um caso de leve agenesia de artéria pulmonar esquerda, associada a cardiopatia isquêmica, diagnosticada por ecocardiografia. Achado pouco frequente na literatura tanto pela localização quanto pela idade do paciente.

Palavras-chave: Pulmonar, Unilateral, Radiografia, Tórax, Agenesia. 


\section{Introducción}

La agenesia unilateral de la arteria pulmonar (AUAP) es una malformación cardiovascular congénita rara, con una prevalencia de 1 de cada 200.000-300.000 individuos. Se postula que es consecuencia de la involución intraútero del sexto arco aórtico, condicionando la ausencia de la parte más proximal de la arteria pulmonar (MuIlor, Ronda, \& Carbonell, 2020, pág. 151). Dicho síndrome fue diagnosticado por primera vez Chevers en 1847, hay enfermos que mueren durante la vida fetal. Comúnmente se asocia con la Tetralogía de Fallot. Se considera una malformación grave con alta mortalidad perinatal (León, Chávez, \& Acevedo, 2019, pág. 33). Es importante señalar que la tasa de mortalidad es mayor cuando la agenesia está en el pulmón derecho, ya que hay una mayor desviación mediastínico, lo que lleva a la compresión traqueal (Pinheiro, de Souza, \& de Oliveira Algeri , 2020, pág. 2).

La agenesia de arteria pulmonar puede diagnosticarse en los primeros años de vida cuando debuta con sintomatología o, como ocurre en la mayor parte de los casos, como hallazgo casual de la radiografía de tórax; descrito en la adolescencia tardía. No existe una clara predilección por el lado derecho o izquierdo, aunque en las diferentes series estudiadas se presenta más frecuentemente en el derecho, siendo las manifestaciones cardiacas menos frecuentes en esta localización (García \& de los Monteros Garde, 2003, pág. 260). Existen dos formas de presentación, una aislada y otra asociada a cardiopatía. Cuando ocurre como una anomalía aislada, los pacientes son generalmente asintomáticos, en cambio, los casos asociados a otras cardiopatías se diagnostican en etapas tempranas de la vida, al presentar con frecuencia síntomas precoces, ya sea por hipoxia, por hipertensión pulmonar y/o insuficiencia cardiaca (Parra-Bravo, y otros, 2002, pág. 162).
Se clasifica en: agenesia, aplasia e hipoplasia, formando parte de las anomalías del desarrollo pulmonar, lo cual se considera un hallazgo infrecuente en la edad adulta. Según varios autores, la prevalencia del género y la etiología difieren; la agenesia pulmonar bilateral llega a ser incompatible con la vida. Su pronóstico va a depender en gran medida si está asociada con otras malformaciones, lo cual puede empeorar presentando un cuadro clínico que puede ir desde la dificultad respiratoria aguda hasta la muerte. Algunos pacientes pueden presentar infecciones pulmonares recurrentes o puede ser un hallazgo incidental; dependiendo de las malformaciones congénitas asociadas y la buena higiene pulmonar (Gradis Santos, Degrandes Rodríguez, Aguilera Tercero, \& Gutiérrez Villanueva, 2021, pág. 78)

Dentro de las malformaciones asociadas a la agenesia pulmonar, 37.5\% son cardiovasculares, como la persistencia del conducto arterioso y el foramen oval; también pueden presentarse otro tipo de anomalías como atresia duodenal, fistula, traqueoesofagica, malformaciones renales y faciales (Lozada \& Burgos, 2018, pág. 47).

\section{Caso clínico}

Paciente masculino 44 años edad, APP rinitis alérgica, AQX septumplastia, AA no refiere, APF no refiere, inmunización Covid 19 SINOVAC 2da dosis. Es trasladado en ambulancia al Hospital Clínica San Francisco con cuadro clínico de 6 horas de evolución, caracterizado por dolor precordial tipo opresivo de gran intensidad que irradia a brazo izquierdo y cuello ipsilateral, además se acompaña de disnea que progresa de medianos a mínimos esfuerzos con náuseas y vomito motivo por el cual ingresa a esta casa hospitalaria para valoración y tratamiento médico especializado. Paciente indica haber tomado en dos ocasiones carga de ácido acetilsalicílico con clopidogrel pero por nauseas las vomito al 
momento de ingreso, el paciente se encuentra despierto orientado en tiempo y espacio y persona afebril, hipotenso con mecánica ventilatoria conservada neuromuscular distal conservado. Los valores iniciales tomados por el personal médico son los siguientes:

- TA: 80/60

- FC: 68

- FR: 18

- SatO2: 97\%

- T: $37 \%$.

\section{Exámenes complementarios}

\section{Radiografía de tórax}

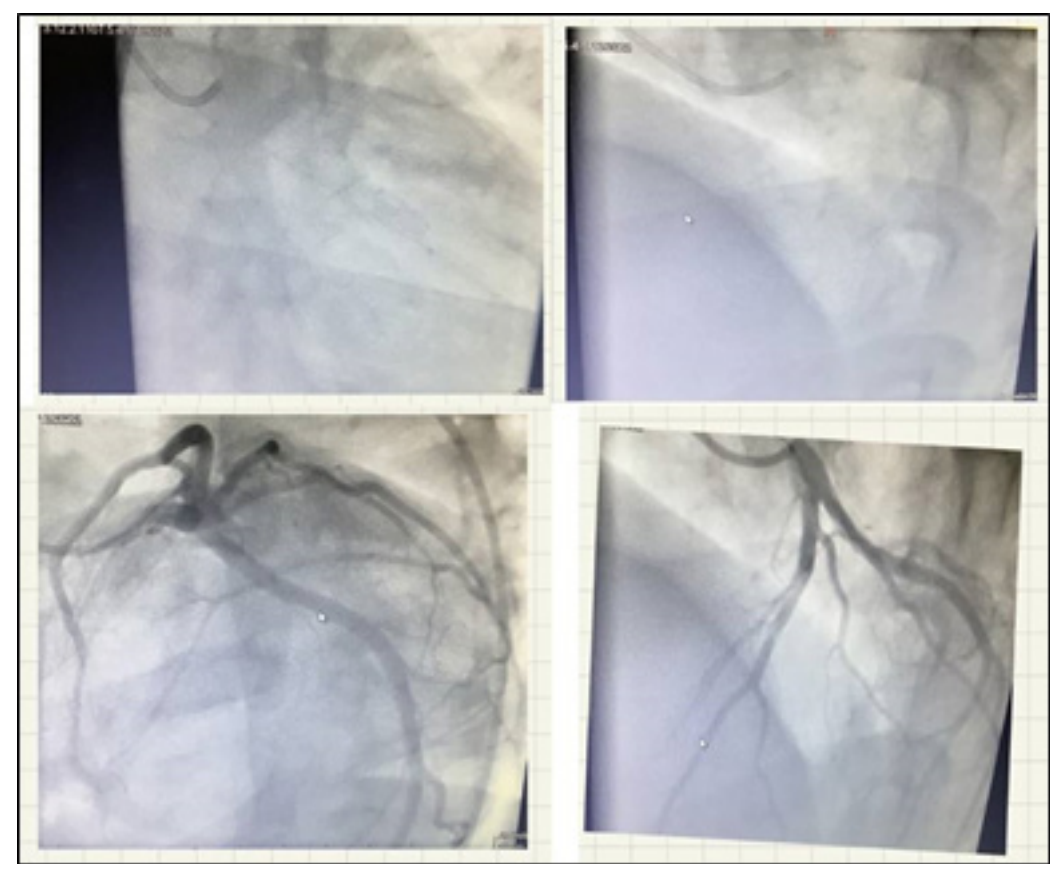

Figura 1. Radiografía de tórax con contraste

Fuente: Los Autores. Tomado de estudios realizados al paciente en el Hospital Clínica San Francisco.

Al paciente se le realiza TAC de tórax con contraste, obteniéndose los siguientes resultados:

- Arteria coronaria derecha de buen calibre, está libre de alteraciones ateromatosas significativas.

- Tronco de arteria coronaria izquierda de buen calibre, libre de obstrucciones.

- Arteria circunfleja de buen calibre, libre de obstrucciones significativas.

- Arteria descendiente anterior de buen calibre, libre de obstrucciones significativas.

\section{Ecocardiografía}




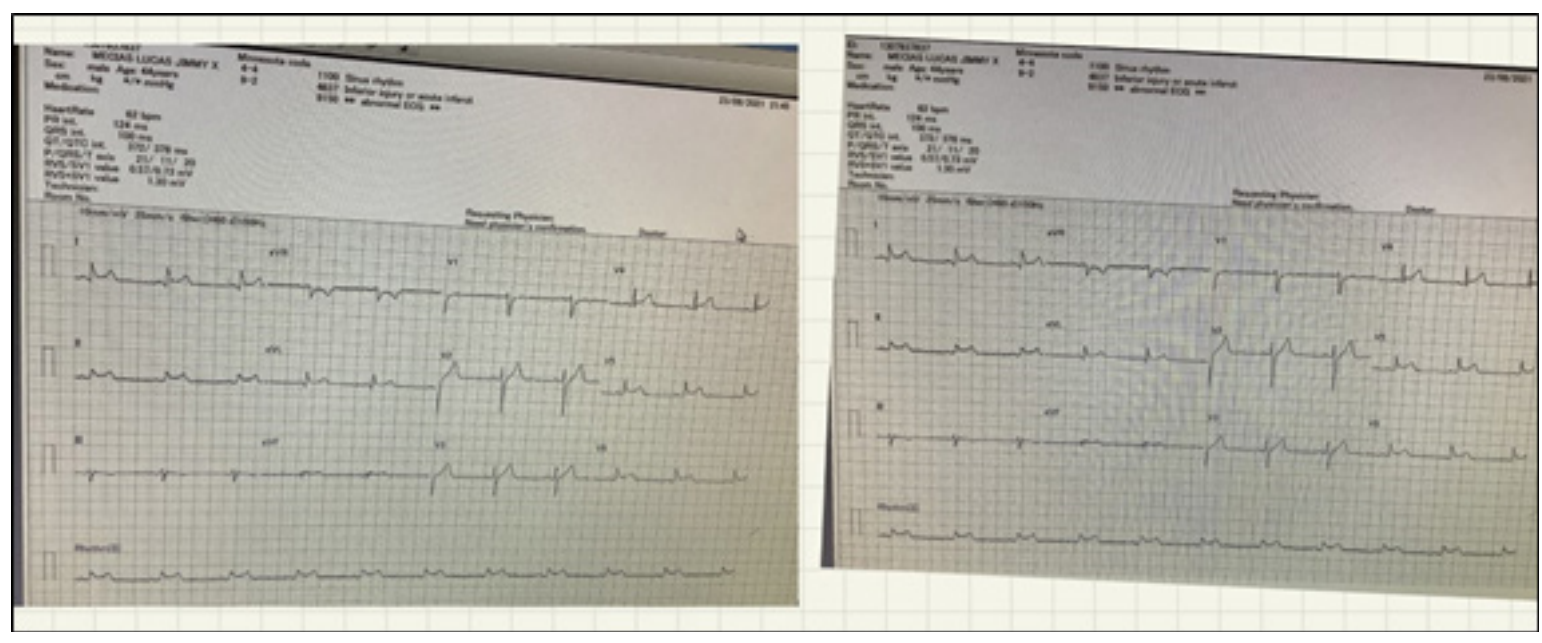

Figura 2. Ecocardiografía MM y Bidimensional

Fuente: Los Autores. Tomado de estudios realizados al paciente en el Hospital Clínica San Francisco.

Los principales hallazgos de la ecocardiografia son los siguientes:

- FSG conservada. FEVI 63-65\%.

- No se evidencian trastornos de la motilidad regional del VI en reposo.

- VI con diámetros en límite de su normalidad, volúmenes y espesores conservados. Al normal.

- Aorta trivalva con adecuada morfología, apertura y cierre de sus sigmoideas coronarias.

- Valvas mitrales con engrosamiento de VAM y presencia prolapso de festón A2 y P3, impresiona elongación de cuerdas tendinosas, sin provocar incompetencia de cierre de sus velos.

- Valvas tricuspideas y pulmonares con adecuada morfología y cinética de sus velos.

- Cavidades cardiacas derechas preservadas. FEVO conservada. TAPSE 27 $\mathrm{mm}$.

- No se evidencia imágenes de trombos, ni masas intracardiacas.

- Pericardio normal. No derrame pericárdicos.

- VCI normal. Colapso inspiratorio mayor de $50 \%$.

- Ritmo sinusal durante todo el estudio.
- La frecuencia cardiaca promedio fue de 63 bpm.

- La frecuencia cardiaca mínima fue de $45 \mathrm{bpm}$.

- La frecuencia cardiaca máxima fue de 109 bpm.

- En total, se encontraron 86816 latidos. Sin pausas mayores de 2.5 segundos y sin trastornos de la conducción AV.

En resumen, paciente hemodinamicamente estable en buenas condiciones clínicas con ecocardiografia que evidencia función sistólica biventricular conservada sin trastornos de la motilidad, holter EKG sin alteraciones, se indica alta médica.

Días después paciente reingresa con trastornos de la oxigenación, con mala mecánica ventilatoria se intuba y se realizan nuevos estudios (Ecocardiografia MM y Bidimensional, Ecocardiograma dopler pulsado y continuo), con los siguientes resultados:

Ecocardiografia MM y Bidimensional

- FSVI deterioro leve FEY 51\% por Simpson BP.

- Movimiento y engrosamiento sistólico, presenta aquinesia anterolateral, ante- 
rior, posteroalteral medial y apical; e hipoquinesia del resto de los segmentos del VI en reposo.

- Diámetros del VI cavidades y espesores conservados.

- Aurícula izquierda de diámetros adecuados.

- Cavidades cardiacas derechas conservadas, FSVD conservada TAPSE 18 $\mathrm{mm}$.

- Aorta trivalva con ecoestructura normal, apertura conservada y cierre de sus velos competente.

- Valvas mitrales y tricuspideas con ecosestructura normal, cinética adecuada de sus velos.

- No trombos, ni masas intracardiacas.

- No se detecta comunicaciones intracardiacas.

- Pericardio normal, no derrame.

- VCl normal, colapso inspiratorio mayor de $50 \%$.

Ecocardiograma doppler, pulsado y continuo.

- Flujo aórtico laminar, no gradiente patológico transalvular, no escape.

- Flujo mitral con patrón trasnmiral de relajación diastólica normal, no se evidencia regurgitación.

- Flujo tricuspideo anterógrado. No gradiente patológico, no se evidencia regurgitación.

- Flujo pulmonar laminar. No gradiente patológico, curva tipo I, no escape, no HTP.

\section{Diagnóstico}

- Disfunción sistólica ventricular izquierda leve, cardiopatía isquémica.

Sin embargo paciente ingresa a terapia intensiva y al siguiente día presenta parada cardiaca, se reanima por 20 minutos, y no se logra retorno a la circulación espontánea y el mismo fallece.

\section{Discusión}

En el presente caso de estudio, se confirma lo evidenciado en otros estudios que la Agenesia de arteria pulmonar es más frecuente en el lado derecho como lo asegura Maggiolo y Rubila (2021) "La agenesia unilateral de la arteria pulmonar (AUAP) es una entidad poco frecuente. Siendo más prevalente la agenesia de la arteria pulmonar derecha (AAPD), la que habitualmente se presenta en forma aislada, es asintomática y subdiagnosticada", por tanto encontrarla del lado izquierdo es poco común, la agenesia de la arteria pulmonar izquierda (AAPI) se asocia a otras malformaciones cardiovasculares, presentando mayor sintomatología. (Maggiolo \& Rubila, 2021).

Sin embargo en las notas iniciales recogidas por el personal médico del Hospital Clínica San Francisco, no hay información de que el paciente haya manifestado la presencia de esta patología durante su vida, por lo que se infiere que no fue diagnosticado, ni presentó alguna sintomatología, hasta que fue trasladado al referido centro asistencial.

En caso de que el paciente se encuentre asintomático, no es necesario administrar tratamiento médico. La principal complicación suelen ser las infecciones respiratorias, en vista de que no se produce un barrido adecuado de las secreciones bronquiales, estas deben ser tratadas precoz y efectivamente con antibióticos (Gradis Santos, Degrandes Rodríguez, Aguilera Tercero, \& Gutiérrez Villanueva, 2021)

La agenesia bilateral implica una falla temprana en el proceso de morfogénesis bronquial y pareciera que el factor de crecimiento de fibroblastos 10 tuviera un importante papel en este estadio, al igual que la deficiencia en su receptor 2 de factor de crecimiento de fibroblastos (FGFR2); claramente la agenesia bilateral es fatal. Esta condición usualmente se diagnostica en la

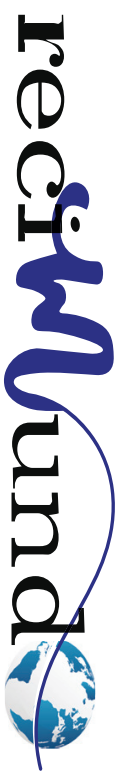


niñez, en vista de que el paciente padece infecciones respiratorias a repetición, aunque una persona puede llegar a la edad adulta sin tener un diagnóstico y con una vida normal si se mantiene asintomática1. (Gradis Santos, Degrandes Rodríguez, Aguilera Tercero, \& Gutiérrez Villanueva, 2021) Lo que confirma que esta patología es infrecuente en la edad adulta como ha sido el caso presentado.

En general el pronóstico no es favorable, se estima un $33 \%$ de mortalidad durante el primer año de vida y el $50 \%$ en los primeros 5 años de vida. Las principales causas de mortalidad son infecciones graves del único pulmón y malformaciones cardiacas y/o de grandes vasos (Gradis Santos, Degrandes Rodríguez, Aguilera Tercero, \& Gutiérrez Villanueva, 2021).

El paciente, en el caso de estudio, se incluye dentro de las estadísticas, confirmando que el pronóstico para esta afección no es favorable, presentando un desenlace mortal luego del diagnóstico.

\section{Conclusiones}

En conclusión, se trató de un caso de agenesia de arteria pulmonar izquierda leve, asociada por una cardiopatía isquémica, diagnosticada por ecocardiograma. Hallazgo infrecuente dentro de la literatura tanto por la localización como por la edad del paciente.

\section{Bibliografía}

García, J. T., \& de los Monteros Garde, M. (2003). AGENESIA DE ARTERIA PULMONAR, TAN FRECUENTE COMO DESCONOCIDA. Neumosur, 15(4), 237-241.

Gradis Santos, O. D., Degrandes Rodríguez, B. J., Aguilera Tercero, L. G., \& Gutiérrez Villanueva, S. J. (2021). AGENESIA PULMONAR COMO HALLAZGO INFRECUENTE EN ADULTOS: REPORTE DE CASO. Revista Científica Ciencia Médica, https://doi.org/10.51581/rccm.v24i1.348.

León, C. E., Chávez, J., \& Acevedo, J. (2019). SINDROME DE AGENESIA VALVULAR PULMONAR CON ORIGEN ANÓMALO DE RAMA PULMONAR IZQUIERDA DE AORTA ASCENDENTE. Revista de Cardiología-Cuerpo Médico Instituto Nacional Cardiovascular INCOR, 5(1), 33-37.

Lozada, A. R., \& Burgos, L. (2018). AGENESIA PULMONAR SEGMENTARIA EN UN PACIENTE PEDIÁTRICO: REPORTE DE CASO CLÍNICO. Revista Médica Basadrina, 12(1), 46-50.

Maggiolo, J., \& Rubila, L. (2021). AGENESIA UNILATERAL DE LA ARTERIA PULMONAR.PRESENTACION DE DOS CASOS Y REVISION DE LA LITERATURA. Neumologia Pediatrica, 16(1), 48-52.

Mullor, M. G., Ronda, V., \& Carbonell, J. (2020). Asimetría radiológica como clave diagnóstica de agenesia de la arteria pulmonar en el adulto. REVISTA DE PATOLOGÍA RESPIRATORIA, 23(4).

Parra-Bravo, J. R., Acosta-Valdez, J., Zepeda-Sanabria, J., Beirana-Palencia, L., Rodríguez-Hernández, L., de Jesús Estrada-Loza, M., \& Vera-Canelo, M. (2002). Agenesia unilateral de una arteria pulmonar y coartación de aorta en un paciente con síndrome de Turner. Revista Mexicana de Cardiología, 13(4), 162-170.

Pinheiro, F. A., de Souza, L., \& de Oliveira Algeri , E. (2020). Agenesia pulmonar direita na unidade de terapia intensiva adulto. Journal of Health \& Biological Sciences, 8(1), 1-5.

\section{CITAR ESTE ARTICULO:}

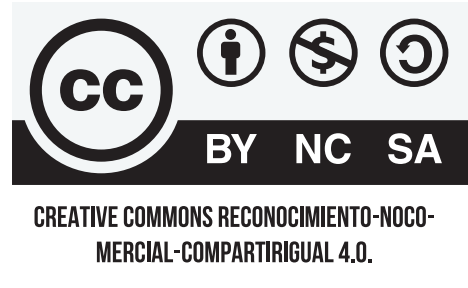

Delgado Zambrano, H. E., Abad Idrovo, M. A., Ledesma Polo, J. A., \& Bohórquez Garces, J. D. (2021). Agenesia de arteria pulmonar. A propósito de un caso en Hospital Clínica San Francisco. RECIMUNDO, 5(4), 200-206. https://

doi.org/10.26820/recimundo/5.(4).oct.2021.200-206 